\title{
REAL HYPERSURFACES OF TYPE $A$ IN COMPLEX TWO-PLANE GRASSMANNIANS RELATED TO THE NORMAL JACOBI OPERATOR
}

\author{
Imsoon Jeong, Young Jin Suh, and Mukut Mani Tripathi
}

\begin{abstract}
In this paper we give a characterization of real hypersurfaces of type $(A)$ in a complex two-plane Grassmannian $G_{2}\left(\mathbb{C}^{m+2}\right)$ which is a tube over a totally geodesic $G_{2}\left(\mathbb{C}^{m+1}\right)$ in $G_{2}\left(\mathbb{C}^{m+2}\right)$, in terms of two commuting conditions related to the normal Jacobi operator and the shape operator.
\end{abstract}

\section{Introduction}

The Jacobi field along geodesics of a given Riemannian manifold $(\bar{M}, \bar{g})$ plays an important role in the study of differential geometry. It satisfies a well-known differential equation. This classical differential equation naturally induces the so-called Jacobi operator. That is, if $\bar{R}$ is the curvature operator of $\bar{M}$, and $X$ is any tangent vector field to $\bar{M}$, the Jacobi operator with respect to $X$ at $p \in \bar{M}, \bar{R}_{X} \in \operatorname{End}\left(T_{p} \bar{M}\right)$, which is defined by

$$
\left(\bar{R}_{X} Y\right)(p)=(\bar{R}(Y, X) X)(p)
$$

for any $Y \in T_{p} \bar{M}$, becomes a self-adjoint endomorphism of the tangent bundle $T \bar{M}$ of $\bar{M}$. Clearly, each tangent vector field $X$ to $\bar{M}$ provides a Jacobi operator with respect to $X$.

The almost contact structure vector fields $\left\{\xi_{1}, \xi_{2}, \xi_{3}\right\}$ are defined by $\xi_{i}=$ - $J_{i} N, i=1,2,3$, where $\left\{J_{1}, J_{2}, J_{3}\right\}$ denotes a canonical local basis of a quaternionic Kaehler structure of $\mathbb{H} P^{m}$ and $N$ a unit normal field of $M$ in $\mathbb{H} P^{m}$. In quaternionic space forms Berndt [2] has introduced the notion of normal Jacobi operator

$$
\bar{R}_{N}=\bar{R}(X, N) N \in \operatorname{End}\left(T_{x} M\right), \quad x \in M
$$

Received December 14, 2010; Revised November 3, 2011.

2010 Mathematics Subject Classification. 53C40, 53C15.

Key words and phrases. real hypersurfaces, Hopf hypersurfaces, normal Jacobi operator, shape operator, commuting conditions.

The first author was supported by grant Proj. No. BSRP-2011-0013381 and the second author by grant Proj. No. BSRP-2011-0025687 from National Research Foundation of Korea. 
for a real hypersurface $M$ in quaternionic projective space $\mathbb{H} P^{m}$ or in quaternionic hyperbolic space $\mathbb{H} H^{m}$, where $\bar{R}$ denotes the curvature tensor of $\mathbb{H} P^{m}$ and $\mathbb{H} H^{m}$ respectively. He [2] has also shown that the curvature-adaptedness, which means that the normal Jacobi operator $\bar{R}_{N}$ commutes with the shape operator $A$, is equivalent to the fact that the distributions $\mathfrak{D}$ and $\mathfrak{D}^{\perp}=$ $\operatorname{Span}\left\{\xi_{1}, \xi_{2}, \xi_{3}\right\}$ are invariant by the shape operator $A$ of $M$, where $T_{x} M=$ $\mathfrak{D} \oplus \mathfrak{D}^{\perp}, x \in M$.

Now let us denote by $G_{2}\left(\mathbb{C}^{m+2}\right)$ the set of all two-dimensional linear subspaces in $\mathbb{C}^{m+2}$, which is said to be a complex two-plane Grassmannian. It is well known that this symmetric space $G_{2}\left(\mathbb{C}^{m+2}\right)$ has both a Kaehler structure $J$ and a quaternionic Kaehler structure $\mathfrak{J}$ not containing $J$. Let $M$ be a real hypersurface in $G_{2}\left(\mathbb{C}^{m+2}\right)$ and $\left\{J_{1}, J_{2}, J_{3}\right\}$ a canonical local basis of $\mathfrak{J}$. Then for a real hypersurface $M$ in $G_{2}\left(\mathbb{C}^{m+2}\right)$ we have the two natural geometric conditions that the 1-dimensional distribution $[\xi]=\operatorname{Span}\{\xi\}$ and both the 3-dimensional distribution $\mathfrak{D}^{\perp}=\operatorname{Span}\left\{\xi_{1}, \xi_{2}, \xi_{3}\right\}$ are invariant under the shape operator $A$ of $M$, where $\xi=-J N, \xi_{\nu}=-J_{\nu} N(\nu=1,2,3)$ and $T_{x} M=\mathfrak{D} \oplus \mathfrak{D}^{\perp}, x \in M$ for a unit normal vector field $N$ of $M$ in $G_{2}\left(\mathbb{C}^{m+2}\right)$ (see [4] and [5]).

By using these two invariant conditions and the result given in Alekseevskii [1], Berndt and Suh [4] proved the following:

Theorem A. Let $M$ be a connected real hypersurface in $G_{2}\left(\mathbb{C}^{m+2}\right), m \geq 3$. Then both $[\xi]$ and $\mathfrak{D}^{\perp}$ are invariant under the shape operator of $M$ if and only if

(A) $M$ is an open part of a tube around a totally geodesic $G_{2}\left(\mathbb{C}^{m+1}\right)$ in $G_{2}\left(\mathbb{C}^{m+2}\right)$, or

(B) $m$ is even, say $m=2 n$, and $M$ is an open part of a tube around a totally geodesic $\mathbb{H} P^{n}$ in $G_{2}\left(\mathbb{C}^{m+2}\right)$.

The Reeb vector field $\xi$ is said to be Hopf if it is invariant under the shape operator $A$. The 1-dimensional foliation of $M$ by the integral manifolds of the Reeb vector field $\xi$ is said to be a Hopf foliation of $M$. We say that $M$ is a Hopf hypersurface in $G_{2}\left(\mathbb{C}^{m+2}\right)$ if and only if the Hopf foliation of $M$ is totally geodesic. By the formulas in Section 3 it can be easily checked that $M$ is Hopf if and only if the Reeb vector field $\xi$ is Hopf.

On the other hand, Lee and Suh [7] gave a characterization of the class of real hypersurfaces of type $(B)$ in $G_{2}\left(\mathbb{C}^{m+2}\right)$ in terms of the Reeb vector field $\xi$ as follows:

Theorem B. Let $M$ be a connected orientable Hopf hypersurface in $G_{2}\left(\mathbb{C}^{m+2}\right)$, $m \geq 3$. Then the Reeb vector field $\xi$ belongs to the distribution $\mathfrak{D}$ if and only if $M$ is locally congruent to an open part of a tube around a totally geodesic $\mathbb{H} P^{n}$ in $G_{2}\left(\mathbb{C}^{m+2}\right)$, where $m=2 n$.

Now in this paper we consider two commuting conditions related to the normal Jacobi operator and the shape operator as follows:

$$
\phi A \bar{R}_{N} X=\bar{R}_{N} \phi A X
$$


for any tangent vector field $X$ on $M$ and

$$
A \phi \phi_{1} X=\phi \phi_{1} A X
$$

for any tangent vector field $X$ on $\mathfrak{D}^{\perp}$, where the structure tensors $\phi$ and $\phi_{1}$ respectively denotes the tangential component on $M$ in $G_{2}\left(\mathbb{C}^{m+2}\right)$ of the Kaehler structure $J$ and the quaternionic Kaehler structure $J_{1}$ (see Section 3 ).

Now let us consider the condition that the Lie derivative coincides with the covariant derivative of the normal Jacobi operator along the direction of the Reeb vector field $\xi$, that is,

$$
\left(\mathcal{L}_{\xi} \bar{R}_{N}\right) X=\left(\nabla_{\xi} \bar{R}_{N}\right) X
$$

for any tangent vector field $X$ on $M$ in $G_{2}\left(\mathbb{C}^{m+2}\right)$. Then it can be easily seen that the two conditions (1.1) and (1.3) are equivalent to each other, because

$$
\left(\mathcal{L}_{\xi} \bar{R}_{N}\right) X=\left(\nabla_{\xi} \bar{R}_{N}\right) X-\phi A \bar{R}_{N} X+\bar{R}_{N} \phi A X
$$

for any vector field $X$ on $M$ in $G_{2}\left(\mathbb{C}^{m+2}\right)$ (see Jeong and Suh [6], page 1119). We give a characterization of real hypersurfaces of type $(A)$ in $G_{2}\left(\mathbb{C}^{m+2}\right)$ as follows:

Main Theorem. Let $M$ be a connected Hopf hypersurface in $G_{2}\left(\mathbb{C}^{m+2}\right), m \geq$ 3 , satisfying (1.1) and (1.2). Then $M$ is locally congruent to an open part of a tube around a totally geodesic $G_{2}\left(\mathbb{C}^{m+1}\right)$ in $G_{2}\left(\mathbb{C}^{m+2}\right)$.

\section{Riemannian geometry of $G_{2}\left(\mathbb{C}^{m+2}\right)$}

In this section we summarize basic material about $G_{2}\left(\mathbb{C}^{m+2}\right)$. For more details we refer to [3], [4] and [5]. By $G_{2}\left(\mathbb{C}^{m+2}\right)$ we denote the set of all complex two-dimensional linear subspaces in $\mathbb{C}^{m+2}$. The special unitary group $G=S U(m+2)$ acts transitively on $G_{2}\left(\mathbb{C}^{m+2}\right)$ with stabilizer isomorphic to $K=S(U(2) \times U(m)) \subset G$. Then $G_{2}\left(\mathbb{C}^{m+2}\right)$ can be identified with the homogeneous space $G / K$, which we equip with the unique analytic structure for which the natural action of $G$ on $G_{2}\left(\mathbb{C}^{m+2}\right)$ becomes analytic. Denote by $\mathfrak{g}$ and $\mathfrak{k}$ the Lie algebra of $G$ and $K$, respectively, and by $\mathfrak{m}$ the orthogonal complement of $\mathfrak{k}$ in $\mathfrak{g}$ with respect to the Cartan-Killing form $B$ of $\mathfrak{g}$. Then $\mathfrak{g}=\mathfrak{k} \oplus \mathfrak{m}$ is an $A d(K)$-invariant reductive decomposition of $\mathfrak{g}$. We put $o=e K$ and identify $T_{o} G_{2}\left(\mathbb{C}^{m+2}\right)$ with $\mathfrak{m}$ in the usual manner. Since $B$ is negative definite on $\mathfrak{g}$, its negative restricted to $\mathfrak{m} \times \mathfrak{m}$ yields a positive definite inner product on $\mathfrak{m}$. By $A d(K)$-invariance of $B$ this inner product can be extended to a $G$-invariant Riemannian metric $g$ on $G_{2}\left(\mathbb{C}^{m+2}\right)$. In this way $G_{2}\left(\mathbb{C}^{m+2}\right)$ becomes a Riemannian homogeneous space, even a Riemannian symmetric space. For computational reasons we normalize $g$ such that the maximal sectional curvature of $\left(G_{2}\left(\mathbb{C}^{m+2}\right), g\right)$ is eight.

When $m=1, G_{2}\left(\mathbb{C}^{3}\right)$ is isometric to the two-dimensional complex projective space $\mathbb{C} P^{2}$ with constant holomorphic sectional curvature eight.

When $m=2$, we note that the isomorphism $\operatorname{Spin}(6) \simeq S U(4)$ yields an isometry between $G_{2}\left(\mathbb{C}^{4}\right)$ and the real Grassmann manifold $G_{2}^{+}\left(\mathbb{R}^{6}\right)$ of oriented 
two-dimensional linear subspaces of $\mathbb{R}^{6}$. So in this paper, we will assume $m \geq 3$ from now on.

The Lie algebra $\mathfrak{k}$ has the direct sum decomposition $\mathfrak{k}=\mathfrak{s} u(m) \oplus \mathfrak{s} u(2) \oplus \mathfrak{R}$, where $\mathfrak{R}$ is the center of $\mathfrak{k}$. Viewing $\mathfrak{k}$ as the holonomy algebra of $G_{2}\left(\mathbb{C}^{m+2}\right)$, the center $\mathfrak{R}$ induces a Kaehler structure $J$ and the $\mathfrak{s} u(2)$-part a quaternionic Kaehler structure $\mathfrak{J}$ on $G_{2}\left(\mathbb{C}^{m+2}\right)$. If $J_{\nu}$ is any almost Hermitian structure in $\mathfrak{J}$, then $J J_{\nu}=J_{\nu} J$, and $J J_{\nu}$ is a symmetric endomorphism with $\left(J J_{\nu}\right)^{2}=I$ and $\operatorname{tr}\left(J J_{\nu}\right)=0, \nu=1,2,3$.

A canonical local basis $J_{1}, J_{2}, J_{3}$ of $\mathfrak{J}$ consists of three local almost Hermitian structures $J_{\nu}$ in $\mathfrak{J}$ such that $J_{\nu} J_{\nu+1}=J_{\nu+2}=-J_{\nu+1} J_{\nu}$, where the index is taken modulo three. Since $\mathfrak{J}$ is parallel with respect to the Riemannian connection $\bar{\nabla}$ of $\left(G_{2}\left(\mathbb{C}^{m+2}\right), g\right)$, there exist for any canonical local basis $J_{1}, J_{2}, J_{3}$ of $\mathfrak{J}$ three local one-forms $q_{1}, q_{2}, q_{3}$ such that

$$
\bar{\nabla}_{X} J_{\nu}=q_{\nu+2}(X) J_{\nu+1}-q_{\nu+1}(X) J_{\nu+2}
$$

for all vector fields $X$ on $G_{2}\left(\mathbb{C}^{m+2}\right)$.

The Riemannian curvature tensor $\bar{R}$ of $G_{2}\left(\mathbb{C}^{m+2}\right)$ is locally given by

$$
\begin{aligned}
\bar{R}(X, Y) Z= & g(Y, Z) X-g(X, Z) Y+g(J Y, Z) J X \\
& -g(J X, Z) J Y-2 g(J X, Y) J Z \\
& +\sum_{\nu=1}^{3}\left\{g(J \nu Y, Z) J_{\nu} X-g\left(J_{\nu} X, Z\right) J_{\nu} Y-2 g\left(J_{\nu} X, Y\right) J_{\nu} Z\right\} \\
& +\sum_{\nu=1}^{3}\left\{g\left(J_{\nu} J Y, Z\right) J_{\nu} J X-g\left(J_{\nu} J X, Z\right) J_{\nu} J Y\right\}
\end{aligned}
$$

where $\left\{J_{1}, J_{2}, J_{3}\right\}$ denotes any canonical local basis of $\mathfrak{J}$ (see [3]).

\section{Some fundamental formulas for real hypersurfaces in $G_{2}\left(\mathbb{C}^{m+2}\right)$}

In this section we derive some basic formulas for a real hypersurface in $G_{2}\left(\mathbb{C}^{m+2}\right)$ (see [9], [10], [11] and [12]).

Let $M$ be a real hypersurface in $G_{2}\left(\mathbb{C}^{m+2}\right)$, that is, a hypersurface in $G_{2}\left(\mathbb{C}^{m+2}\right)$ with real codimension one. The induced Riemannian metric on $M$ will also be denoted by $g$, and $\nabla$ denotes the Riemannian connection of $(M, g)$. Let $N$ be a local unit normal vector field of $M$ and $A$ the shape operator of $M$ with respect to $N$.

Now let us put

$$
J X=\phi X+\eta(X) N, \quad J_{\nu} X=\phi_{\nu} X+\eta_{\nu}(X) N
$$

for any tangent vector field $X$ of a real hypersurface $M$ in $G_{2}\left(\mathbb{C}^{m+2}\right)$, where $N$ denotes a unit normal vector field of $M$ in $G_{2}\left(\mathbb{C}^{m+2}\right)$. From the Kähler structure $J$ of $G_{2}\left(\mathbb{C}^{m+2}\right)$ there exists an almost contact metric structure $(\phi, \xi, \eta, g)$ 
induced on $M$ in such a way that

$$
\phi^{2} X=-X+\eta(X) \xi, \quad \eta(\xi)=1, \quad \phi \xi=0, \quad \eta(X)=g(X, \xi)
$$

for any vector field $X$ on $M$. Furthermore, let $\left\{J_{1}, J_{2}, J_{3}\right\}$ be a canonical local basis of $\mathfrak{J}$. Then the quaternionic Kähler structure $J_{\nu}$ of $G_{2}\left(\mathbb{C}^{m+2}\right)$, together with the condition $J_{\nu} J_{\nu+1}=J_{\nu+2}=-J_{\nu+1} J_{\nu}$ in Section 1, induce an almost contact metric 3 -structure $\left(\phi_{\nu}, \xi_{\nu}, \eta_{\nu}, g\right)$ on $M$ as follows:

$$
\begin{aligned}
& \phi_{\nu}^{2} X=-X+\eta_{\nu}(X) \xi_{\nu}, \quad \eta_{\nu}\left(\xi_{\nu}\right)=1, \quad \phi_{\nu} \xi_{\nu}=0 \\
& \phi_{\nu+1} \xi_{\nu}=-\xi_{\nu+2}, \quad \phi_{\nu} \xi_{\nu+1}=\xi_{\nu+2}, \\
& \phi_{\nu} \phi_{\nu+1} X=\phi_{\nu+2} X+\eta_{\nu+1}(X) \xi_{\nu}, \\
& \phi_{\nu+1} \phi_{\nu} X=-\phi_{\nu+2} X+\eta_{\nu}(X) \xi_{\nu+1}
\end{aligned}
$$

for any vector field $X$ tangent to $M$. Moreover, from the commuting property of $J_{\nu} J=J J_{\nu}, \nu=1,2,3$ in Section 1 and (3.1), the relation between these two almost contact metric structures $(\phi, \xi, \eta, g)$ and $\left(\phi_{\nu}, \xi_{\nu}, \eta_{\nu}, g\right), \nu=1,2,3$, can be given by

$$
\begin{aligned}
& \phi \phi_{\nu} X=\phi_{\nu} \phi X+\eta_{\nu}(X) \xi-\eta(X) \xi_{\nu} \\
& \eta_{\nu}(\phi X)=\eta\left(\phi_{\nu} X\right), \quad \phi \xi_{\nu}=\phi_{\nu} \xi .
\end{aligned}
$$

On the other hand, from the Kähler structure $J$, that is, $\bar{\nabla} J=0$ and the quaternionic Kähler structure $J_{\nu}$, together with Gauss and Weingarten equations it follows that

$$
\begin{gathered}
\left(\nabla_{X} \phi\right) Y=\eta(Y) A X-g(A X, Y) \xi, \quad \nabla_{X} \xi=\phi A X \\
\nabla_{X} \xi_{\nu}=q_{\nu+2}(X) \xi_{\nu+1}-q_{\nu+1}(X) \xi_{\nu+2}+\phi_{\nu} A X \\
\left(\nabla_{X} \phi_{\nu}\right) Y=- \\
-q_{\nu+1}(X) \phi_{\nu+2} Y+q_{\nu+2}(X) \phi_{\nu+1} Y+\eta_{\nu}(Y) A X \\
-g(A X, Y) \xi_{\nu} .
\end{gathered}
$$

Summing up these formulas, we find the following:

$$
\begin{aligned}
\nabla_{X}\left(\phi_{\nu} \xi\right)= & \nabla_{X}\left(\phi \xi_{\nu}\right) \\
= & \left(\nabla_{X} \phi\right) \xi_{\nu}+\phi\left(\nabla_{X} \xi_{\nu}\right) \\
= & q_{\nu+2}(X) \phi_{\nu+1} \xi-q_{\nu+1}(X) \phi_{\nu+2} \xi+\phi_{\nu} \phi A X \\
& -g(A X, \xi) \xi_{\nu}+\eta\left(\xi_{\nu}\right) A X
\end{aligned}
$$


Using the above expression (2.2) for the curvature tensor $\widetilde{R}$ of $G_{2}\left(\mathbb{C}^{m+2}\right)$, the equation of Codazzi becomes:

(3.8)

$$
\begin{aligned}
\left(\nabla_{X} A\right) Y-\left(\nabla_{Y} A\right) X= & \eta(X) \phi Y-\eta(Y) \phi X-2 g(\phi X, Y) \xi \\
& +\sum_{\nu=1}^{3}\left\{\eta_{\nu}(X) \phi_{\nu} Y-\eta_{\nu}(Y) \phi_{\nu} X-2 g\left(\phi_{\nu} X, Y\right) \xi_{\nu}\right\} \\
& +\sum_{\nu=1}^{3}\left\{\eta_{\nu}(\phi X) \phi_{\nu} \phi Y-\eta_{\nu}(\phi Y) \phi_{\nu} \phi X\right\} \\
& +\sum_{\nu=1}^{3}\left\{\eta(X) \eta_{\nu}(\phi Y)-\eta(Y) \eta_{\nu}(\phi X)\right\} \xi_{\nu} .
\end{aligned}
$$

\section{Key lemmas}

Now we consider the commuting condition (1.1) related to the normal Jacobi operator.

The normal Jacobi operator $\bar{R}_{N}$ of the unit normal vector field $N$ can be defined from the curvature tensor $\bar{R}$ of the ambient space $G_{2}\left(\mathbb{C}^{m+2}\right)$. By putting $Y=Z=N$ for the unit normal vector $N$ in (2.2), we calculate the normal Jacobi operator $\bar{R}_{N}$ in such a way that

$$
\begin{aligned}
\bar{R}_{N} X= & \bar{R}(X, N) N \\
= & X+3 \eta(X) \xi+3 \sum_{\nu=1}^{3} \eta_{\nu}(X) \xi_{\nu} \\
& -\sum_{\nu=1}^{3}\left\{\eta_{\nu}(\xi)\left(\phi_{\nu} \phi X-\eta(X) \xi_{\nu}\right)-\eta_{\nu}(\phi X) \phi_{\nu} \xi\right\}
\end{aligned}
$$

for any tangent vector field $X$ on $M$ in $G_{2}\left(\mathbb{C}^{m+2}\right)([8])$.

By (1.1) and (4.1), a Hopf hypersurface $M$ in $G_{2}\left(\mathbb{C}^{m+2}\right)$ with commuting normal Jacobi operator as in (1.1) satisfies the following

$$
\begin{aligned}
& 3 \sum_{\nu=1}^{3} \eta_{\nu}(X) \phi A \xi_{\nu}-\sum_{\nu=1}^{3}\left\{\eta_{\nu}(\xi)\left(\phi A \phi_{\nu} \phi X-\eta(X) \phi A \xi_{\nu}\right)-\eta_{\nu}(\phi X) \phi A \phi_{\nu} \xi\right\} \\
= & 3 \sum_{\nu=1}^{3} \eta_{\nu}(\phi A X) \xi_{\nu}-\sum_{\nu=1}^{3}\left\{-\eta_{\nu}(\xi) \phi_{\nu} A X+\eta_{\nu}(A X) \phi_{\nu} \xi\right\}
\end{aligned}
$$

for any tangent vector field $X$ on $M$ in $G_{2}\left(\mathbb{C}^{m+2}\right)$. From $(4.2)$, we assert the following:

Lemma 4.1. Let $M$ be a connected Hopf hypersurface in $G_{2}\left(\mathbb{C}^{m+2}\right), m \geq 3$. If $M$ satisfies (1.1) and (1.2), then the Reeb vector field $\xi$ belongs to either the distribution $\mathfrak{D}$ or the distribution $\mathfrak{D}^{\perp}$. 
Proof. In order to prove our lemma, let us put $\xi=\eta\left(X_{0}\right) X_{0}+\eta\left(\xi_{1}\right) \xi_{1}$ for some unit $X_{0} \in \mathfrak{D}$ and $\eta\left(X_{0}\right) \eta\left(\xi_{1}\right) \neq 0$. And by putting $X=\xi$ in (4.2), we have

$$
\sum_{\nu=1}^{3} \eta_{\nu}(\xi) \phi A \xi_{\nu}=0 .
$$

From this, we have $\eta_{1}(\xi) \phi A \xi_{1}=0$. Since $\eta_{1}(\xi) \neq 0$, we obtain

$$
\phi A \xi_{1}=0 .
$$

Then from this, by applying $\phi$ we have for some unit $X_{0} \in \mathfrak{D}$

$$
A \xi_{1}=\alpha \eta\left(\xi_{1}\right) \eta\left(X_{0}\right) X_{0}+\alpha \eta^{2}\left(\xi_{1}\right) \xi_{1},
$$

where $\alpha=g(A \xi, \xi)$.

On the other hand, from the condition (1.2), by putting $X=\xi_{1}$ we have $0=\phi \phi_{1} A \xi_{1}$. Then, by using (3.3) and (4.3), we obtain $0=\eta_{1}\left(A \xi_{1}\right) \xi-\eta\left(A \xi_{1}\right) \xi_{1}$. From this, taking an inner product with $\xi_{1}$, and using the expression $\xi=$ $\eta\left(X_{0}\right) X_{0}+\eta\left(\xi_{1}\right) \xi_{1}$ gives $\eta_{1}\left(A \xi_{1}\right) \eta\left(\xi_{1}\right) \xi_{1}=\eta\left(A \xi_{1}\right) \xi_{1}$. Then from the assumption that $M$ is Hopf we have the following

$$
\alpha=\eta_{1}\left(A \xi_{1}\right) .
$$

By taking an inner product (4.4) with $\xi_{1}$ and using (4.5), we have

$$
\alpha\left(1-\eta^{2}\left(\xi_{1}\right)\right)=0 .
$$

When $\alpha=0$, we can differentiate $A \xi=0$. Then by a theorem due to Berndt and Suh (see [5], page 92) we know that

$$
\sum_{\nu=1}^{3} \eta_{\nu}(\xi) \phi \xi_{\nu}=0
$$

This also gives $\xi \in \mathfrak{D}$ or $\xi \in \mathfrak{D}^{\perp}$.

Next we consider the case that the function $\alpha$ is non-vanishing. Then from (4.6) we have $\eta^{2}\left(\xi_{1}\right)=1$. This means $\xi \in \mathfrak{D}^{\perp}$, which gives a contradiction. Accordingly, we get a complete proof of our lemma.

For the case that the Reeb vector field $\xi$ belongs to the distribution $\mathfrak{D}$, we refer to a lemma due to Lee and Suh [7] as follows:

Lemma 4.2. Let $M$ be a connected Hopf hypersurface in $G_{2}\left(\mathbb{C}^{m+2}\right), m \geq 3$. If $\xi \in \mathfrak{D}$, then $g\left(A \mathfrak{D}, \mathfrak{D}^{\perp}\right)=0$.

Now in the case that $\xi$ belongs to the distribution $\mathfrak{D}^{\perp}$, we obtain the following

Lemma 4.3. Let $M$ be a connected Hopf hypersurface in $G_{2}\left(\mathbb{C}^{m+2}\right), m \geq 3$, satisfying (1.1) and (1.2). If $\xi \in \mathfrak{D}^{\perp}$, then $g\left(A \mathfrak{D}, \mathfrak{D}^{\perp}\right)=0$. 
Proof. We will show that $g\left(A X, \xi_{\nu}\right)=0$ for any $\nu=1,2,3$ and any $X \in \mathfrak{D}$. In order to do this, we may put $\xi=\xi_{1}$, because $\xi \in \mathfrak{D}^{\perp}$. Then it suffices to show that $\eta_{2}(A X)=\eta_{3}(A X)=0$ for any $X \in \mathfrak{D}$.

From (4.2), we have

$$
\begin{aligned}
& 3 \sum_{\nu=1}^{3} \eta_{\nu}(X) \phi A \xi_{\nu}-\phi A \phi_{1} \phi X+\sum_{\nu=1}^{3} \eta_{\nu}(\phi X) \phi A \phi_{\nu} \xi \\
= & 3 \sum_{\nu=1}^{3} \eta_{\nu}(\phi A X) \xi_{\nu}-\sum_{\nu=1}^{3} \eta_{\nu}(A X) \phi_{\nu} \xi+\phi_{1} A X,
\end{aligned}
$$

where we have used the formula (1.1). Then, by applying $\phi$ to both sides of (4.7), we have

$$
\begin{aligned}
& -2 \eta_{2}(X) A \xi_{2}-2 \eta_{3}(X) A \xi_{3}+A \phi_{1} \phi X \\
= & -2 \eta_{3}(A X) \xi_{3}-2 \eta_{2}(A X) \xi_{2}+\phi \phi_{1} A X
\end{aligned}
$$

for any tangent vector field $X$ on $M$.

By putting $X=\xi_{2}$ in (4.8), and using (1.2) and (3.3), we get $A \xi_{2}=$ $\eta_{2}\left(A \xi_{2}\right) \xi_{2}+\eta_{3}\left(A \xi_{2}\right) \xi_{3}$. From this, by taking an inner product with any $X \in \mathfrak{D}$, we get

$$
g\left(A X, \xi_{2}\right)=0 .
$$

Similarly, putting $X=\xi_{3}$ in (4.8), and using (1.2) and (3.3), we get $A \xi_{3}=$ $\eta_{2}\left(A \xi_{3}\right) \xi_{2}+\eta_{3}\left(A \xi_{3}\right) \xi_{3}$. Then for any $X \in \mathfrak{D}$, we get

$$
g\left(A X, \xi_{3}\right)=0 .
$$

Hence, from (4.9) and (4.10), it follows that $g\left(A X, \xi_{\nu}\right)=0$ for $\nu=1,2,3$ and any $X \in \mathfrak{D}$, that is, $g\left(A \mathfrak{D}, \mathfrak{D}^{\perp}\right)=0$. This gives a complete proof of our lemma.

\section{Proof of Main Theorem}

Summing up Lemmas 4.1, 4.2 and 4.3, and Theorems A and B in the introduction, we know that any connected Hopf hypersurface in $G_{2}\left(\mathbb{C}^{m+2}\right)$ satisfying conditions (1.1) and (1.2) is congruent to one either of type $(A)$ or type $(B)$. Now conversely, let us check whether real hypersurfaces of type $(A)$ or type $(B)$ in $G_{2}\left(\mathbb{C}^{m+2}\right)$ satisfy both conditions (1.1) and (1.2).

Now we recall the following proposition due to Berndt and Suh [4] as follows:

Proposition A. Let $M$ be a connected real hypersurface of $G_{2}\left(\mathbb{C}^{m+2}\right)$. Suppose that $A \mathfrak{D} \subset \mathfrak{D}, A \xi=\alpha \xi$, and $\xi$ is tangent to $\mathfrak{D}^{\perp}$. Let $J_{1} \in \mathfrak{J}$ be the almost Hermitian structure such that $J N=J_{1} N$. Then $M$ has three $($ if $r=\pi / 2 \sqrt{8})$ or four (otherwise) distinct constant principal curvatures

$$
\alpha=\sqrt{8} \cot (\sqrt{8} r), \quad \beta=\sqrt{2} \cot (\sqrt{2} r), \quad \lambda=-\sqrt{2} \tan (\sqrt{2} r), \quad \mu=0
$$

with some $r \in(0, \pi / \sqrt{8})$. The corresponding multiplicities are

$$
m(\alpha)=1, \quad m(\beta)=2, \quad m(\lambda)=2 m-2=m(\mu),
$$


and the corresponding eigenspaces are

$$
\begin{aligned}
& T_{\alpha}=\mathbb{R} \xi=\mathbb{R} J N=\mathbb{R} \xi_{1}=\operatorname{Span}\{\xi\}=\operatorname{Span}\left\{\xi_{1}\right\}, \\
& T_{\beta}=\mathbb{C}^{\perp} \xi=\mathbb{C}^{\perp} N=\mathbb{R} \xi_{2} \oplus \mathbb{R} \xi_{3}=\operatorname{Span}\left\{\xi_{2}, \xi_{3}\right\}, \\
& T_{\lambda}=\left\{X \mid X \perp \mathbb{H} \xi, J X=J_{1} X\right\}, \\
& T_{\mu}=\left\{X \mid X \perp \mathbb{H} \xi, J X=-J_{1} X\right\},
\end{aligned}
$$

where $\mathbb{R} \xi, \mathbb{C} \xi$ and $\mathbb{H} \xi$ respectively denotes real, complex and quaternionic span of the structure vector field $\xi$ and $\mathbb{C}^{\perp} \xi$ denotes the orthogonal complement of $\mathbb{C} \xi$ in $\mathbb{H} \xi$.

As the first part, we may check that real hypersurfaces of type $(A)$ satisfy the condition (1.1). Using Proposition A and (4.7), it can be easily checked that such hypersurfaces satisfy the formula (1.1) as follows:

Case 1-1. $X=\xi=\xi_{1}$

In this case it can be easily checked that the two sides are equal to each other.

Case 1-2. $X=\xi_{2}, \xi_{3}$

Then we put $A \xi_{2}=\beta \xi_{2}, A \xi_{3}=\beta \xi_{3}$, where $\beta=\sqrt{2} \cot (\sqrt{2} r)$. Then by putting $X=\xi_{2}$ in (4.7) we have

$$
3 \phi A \xi_{2}-\beta \phi \xi_{2}-\phi A \phi_{3} \xi=-3 \beta \xi_{3}+\beta \phi_{1} \xi_{2}-\beta \phi_{2} \xi \text {. }
$$

From this we know that both sides are equal to $-\beta \xi_{3}$. Similarly, by putting $X=\xi_{3}$ in (4.7) we know that both sides are equal to $\beta \xi_{2}$.

Case 1-3. $X \in T_{\lambda}=\left\{X \mid X \perp \mathbb{H} \xi, \phi X=\phi_{1} X\right\}$

By putting $X \in T_{\lambda}, \lambda=-\sqrt{2} \tan (\sqrt{2} r)$ in (4.7), we get $-\phi A \phi_{1} \phi X=\phi_{1} A X$. From this we deduce that the both sides are equal to $\lambda \phi X$.

Case 1-4. $X \in T_{\mu}=\left\{X \mid X \perp \mathbb{H} \xi, \phi X=-\phi_{1} X\right\}$

By putting $X \in T_{\mu}, \mu=0$ in (4.7), we get $-\phi A \phi_{1} \phi X=\phi_{1} A X$. From this we deduce that they are equal to $\mu \phi_{1} X$.

Next, let us check whether real hypersurfaces of type $(A)$ satisfy also the condition (1.2).

Case 2-1. $X=\xi=\xi_{1}$

In this case the two sides are equal to each other.

Case 2-2. $X=\xi_{2}$

Then we put $A \xi_{2}=\beta \xi_{2}$, where $\beta=\sqrt{2} \cot (\sqrt{2} r)$. Then by putting $X=\xi_{2}$ in (1.2), we find that both sides are equal to $\beta \xi_{2}$.

Case 2-3. $X=\xi_{3}$ 
By putting $X=\xi_{3}$ in (1.2), we find that they are equal to $\beta \xi_{3}$.

Thus by using Proposition A, we see that hypersurfaces of type $(A)$ in $G_{2}\left(\mathbb{C}^{m+2}\right)$ satisfy the formulas (1.1) and (1.2), respectively.

From this, we may assert the following:

Theorem 5.1. Let $M$ be a connected Hopf hypersurface in $G_{2}\left(\mathbb{C}^{m+2}\right), m \geq 3$, satisfying two commuting conditions (1.1) and (1.2). If the Reeb vector field $\xi$ belongs to the distribution $\mathfrak{D}^{\perp}$, then $M$ is locally congruent to an open part of a tube around a totally geodesic $G_{2}\left(\mathbb{C}^{m+1}\right)$ in $G_{2}\left(\mathbb{C}^{m+2}\right)$.

Now as a second part, we may check whether real hypersurfaces of type $(B)$ satisfy the conditions (1.1) and (1.2). In order to do this we apply a proposition due to Berndt and Suh [4] as follows:

Proposition B. Let $M$ be a connected real hypersurface of $G_{2}\left(\mathbb{C}^{m+2}\right)$. Suppose that $A \mathfrak{D} \subset \mathfrak{D}, A \xi=\alpha \xi$, and $\xi$ is tangent to $\mathfrak{D}$. Then the quaternionic dimension $m$ of $G_{2}\left(\mathbb{C}^{m+2}\right)$ is even, say $m=2 n$, and $M$ has five distinct constant principal curvatures

$$
\alpha=-2 \tan (2 r), \quad \beta=2 \cot (2 r), \quad \gamma=0, \quad \lambda=\cot (r), \quad \mu=-\tan (r)
$$

with some $r \in(0, \pi / 4)$. The corresponding multiplicities are

$$
m(\alpha)=1, \quad m(\beta)=3=m(\gamma), \quad m(\lambda)=4 n-4=m(\mu)
$$

and the corresponding eigenspaces are

$$
\begin{aligned}
& T_{\alpha}=\mathbb{R} \xi=\operatorname{Span}\{\xi\}, \\
& T_{\beta}=\mathfrak{J} J \xi=\operatorname{Span}\left\{\xi_{\nu} \mid \nu=1,2,3\right\}, \\
& T_{\gamma}=\mathfrak{J} \xi=\operatorname{Span}\left\{\phi_{\nu} \xi \mid \nu=1,2,3\right\}, \\
& T_{\lambda}, \quad T_{\mu},
\end{aligned}
$$

where

$$
T_{\lambda} \oplus T_{\mu}=(\mathbb{H} \mathbb{C} \xi)^{\perp}, \quad \mathfrak{J} T_{\lambda}=T_{\lambda}, \quad \mathfrak{J} T_{\mu}=T_{\mu}, \quad J T_{\lambda}=T_{\mu} .
$$

Now let us suppose that a real hypersurface $M$ of type $(B)$ satisfies the condition (1.1). Then from (4.2) it follows that

$$
\begin{aligned}
& 3 \sum_{\nu=1}^{3} \eta_{\nu}(X) \phi A \xi_{\nu}+\sum_{\nu=1}^{3} \eta_{\nu}(\phi X) \phi A \phi_{\nu} \xi \\
= & 3 \sum_{\nu=1}^{3} \eta_{\nu}(\phi A X) \xi_{\nu}-\sum_{\nu=1}^{3} \eta_{\nu}(A X) \phi_{\nu} \xi
\end{aligned}
$$

for any tangent vector field $X$ on $M$ in $G_{2}\left(\mathbb{C}^{m+2}\right)$. From this, by putting $X=\xi_{2}$, we have $\beta \xi_{3}=0$.

Thus, we have $\beta=2 \cot (2 r)=0$ and this case can not occur for any $r \in$ $(0, \pi / 4)$ in Proposition B. Accordingly, we know that real hypersurfaces of type $(B)$ do not satisfy the condition (1.1).

Next let us check whether real hypersurfaces of type $(B)$ in $G_{2}\left(\mathbb{C}^{m+2}\right)$ satisfy condition (1.2) or not when the Reeb vector field $\xi$ belongs to the distribution 
D. By putting $X=\xi_{2}$ in (1.2) and using Proposition $\mathrm{B}$, we have $\beta \phi \xi_{3}=0$, which gives $\beta=0$. But in Proposition $\mathrm{B}$ we know that $\beta=2 \cot 2 r$. Thus, we have $\beta=2 \cot (2 r)=0$ and this case also can not occur for any $r \in$ $(0, \pi / 4)$. Hence we conclude that real hypersurfaces of type $(B)$ do not satisfy the condition (1.2).

Thus we assert the following:

Theorem 5.2. There does not exist any connected Hopf hypersurface in $G_{2}\left(\mathbb{C}^{m+2}\right), m \geq 3$, satisfying (1.1) and (1.2) provided the Reeb vector field $\xi$ belongs to the distribution $\mathfrak{D}$.

Therefore, summing up Lemma 4.1, Theorems 5.1 and 5.2, we complete the proof of our Main Theorem in Introduction.

Remark. In Section 1 we have shown that the conditions (1.1) and (1.3) are equivalent to each other. Accordingly, we want to remark here that a connected Hopf hypersurface $M$ in complex two-plane Grassmannian $G_{2}\left(\mathbb{C}^{m+2}\right)$ satisfying $(1.2)$ and $(1.3)$ is also congruent to a tube over a totally geodesic $G_{2}\left(\mathbb{C}^{m+1}\right)$ in $G_{2}\left(\mathbb{C}^{m+2}\right)$.

Acknowledgments. The authors would like to express their sincere gratitude to the referee for his careful reading of our manuscript and useful suggestions to develop the first version of this paper.

\section{References}

[1] D. V. Alekseevskii, Compact quaternion spaces, Func. Anal. Appl. 2 (1966), 106-114.

[2] J. Berndt, Real hypersurfaces in quaternionic space forms, J. Reine Angew. Math. 419 (1991), 9-26.

[3] _ Riemannian geometry of complex two-plane Grassmannians, Rend. Sem. Mat. Univ. Politec. Torino 55 (1997), no. 1, 19-83.

[4] J. Berndt and Y. J. Suh, Real hypersurfaces in complex two-plane Grassmannians, Monatsh. Math. 127 (1999), no. 1, 1-14.

[5] _ Real hypersurfaces with isometric Reeb flows in complex two-plane Grassmannians, Monatsh. Math. 137 (2002), no. 2, 87-98.

[6] I. Jeong and Y. J. Suh, Real hypersurfaces in complex two-plane Grassmannians with Lie $\xi$-parallel normal Jacobi operator, J. Korean Math. Soc. 45 (2008), no. 4, 1113-1133.

[7] H. Lee and Y. J. Suh, Real hypersurfaces of type B in complex two-plane Grassmannians related to the Reeb vector, Bull. Korean Math. Soc. 47 (2010), no. 3, 551-561.

[8] J. D. Pérez, I. Jeong, and Y. J. Suh, Real hypersurfaces in complex two-plane Grassmannians with commuting normal Jacobi operator, Acta Math. Hungar. 117 (2007), no. 3, 201-217.

[9] Y. J. Suh, Real hypersurfaces in complex two-plane Grassmannians with parallel shape operator, Bull. Austral. Math. Soc. 67 (2003), no. 3, 493-502.

[10] _ Real hypersurfaces in complex two-plane Grassmannians with parallel shape operator II, J. Korean Math. Soc. 41 (2004), no. 3, 535-565.

[11] _ Real hypersurfaces in complex two-plane Grassmannians with vanishing Lie derivatives, Canad. Math. Bull. 49 (2006), no. 1, 134-143.

[12] Real hypersurfaces of type B in complex two-plane Grassmannians, Monatsh. Math. 147 (2006), no. 4, 337-355. 
IMSOON JEONG

Department of Mathematics

KYUNGPOOK NATIONAL University

TAEGU 702-701, Korea

E-mail address: imsoon.jeong@gmail.com

YOUNG JIN SUH

Department of Mathematics

KYUNGPOOK NATIONAL UNIVERSITY

TAEGU 702-701, KoreA

E-mail address: yjsuh@knu.ac.kr

Mukut Mani Tripathi

Department of Mathematics AND

DST CENTRE FOR INTERDISCIPLINARY

Mathematical Sciences

BARANAS Hindu UNIVERSITy

VARANASI 221005, INDIA

E-mail address: mmtripathi66@yahoo.com 